\title{
Evaluación físico-química de aceite pigmentado obtenido de la cabeza de camarón.
}

\author{
By J.A. Núñez-Gastélum ${ }^{1}$, D.I. Sánchez-Machado ${ }^{1}$, J. López-Cervantes ${ }^{1 *}$, P. Paseiro-Losada ${ }^{2}$, \\ R. Sendón ${ }^{2}$, A.T. Sanches-Silva ${ }^{3}$, H.S. Costa ${ }^{3}$, G.P. Aurrekoetxea ${ }^{4}$, I. Angulo ${ }^{4}$ y H. Soto-Valdez ${ }^{5}$
}

\author{
1Departamento de Biotecnología y Ciencias Alimentarias, Instituto Tecnológico de Sonora, \\ Cd. Obregón, MX-85000, Sonora, México. \\ ${ }^{2}$ Departamento de Química Analítica, Nutrición y Ciencia de los Alimentos, Facultad de Farmacia, \\ Universidad de Santiago de Compostela, Santiago de Compostela, E-15782, España. \\ ${ }^{3}$ Departamento de Alimentação e Nutrição, Instituto Nacional de Saúde Doutor Ricardo Jorge, \\ I.P., 1649-016, Lisboa, Portugal. \\ ${ }^{4}$ Centro Tecnológico Gaiker, Parque Tecnológico, 48170 Zamudio, España. \\ ${ }^{5}$ Coordinación de Tecnología de Alimentos de Origen Vegetal, Centro de investigación \\ en Alimentación y Desarrollo, A.C., Hermosillo, MX-83000, Sonora, México. \\ ( ${ }^{\star}$ Autor para la correspondencia: jaime.lopez@itson.edu.mx)
}

\section{RESUMEN}

Evaluación físico-química de aceite pigmentado obtenido de la cabeza de camarón.

En este trabajo se presenta el análisis proximal, caracterización físico-química, perfil de ácidos grasos y contenido de astaxantina en aceite pigmentado aislado por fermentación láctica de los residuos de camarón. Los lípidos son los componentes mayoritarios (95\%). El índice de saponificación es $178.62 \mathrm{mg} \mathrm{KOH} / \mathrm{g}$, el de yodo $139.8 \mathrm{cg}$ yodo/g, y los peróxidos no fueron detectados. La densidad y la viscosidad fueron de $0.92 \mathrm{mg} / \mathrm{ml}$ y 64 centipoises, respectivamente. Los ácidos grasos en mayor cantidad fueron el linoleico (C18:2n6), oleico (C18:1n9) y palmítico (C16:0). El ácido eicosapentaenoico (C20:5n3, EPA) y el docosahexaenoico (C22:6n3, DHA) suman el 9\% del total. El contenido promedio de astaxantina fue de $2.72 \mathrm{mg} / \mathrm{g}$ base seca. El aceite pigmentado es una fuente dietética de nutrientes con alto valor como la astaxantina.

PALABRAS CLAVE: Aceite - Ácidos grasos - Astaxantina - Cabeza de camarón - Cromatografía de gases - Fermentación láctica.

\section{SUMMARY}

Physical and chemical properties of pigmented oil obtained from shrimp heads.

In this work the proximal analysis, physicochemical characterization, fatty acid profile and astaxanthin content of pigmented oil obtained by fermentation shrimp heads are presented. Lipids are the major components in the oil (95\%). The saponification number is $178.62 \mathrm{mg}$ $\mathrm{KOH} / \mathrm{g}$, iodine value $139.8 \mathrm{cg}$ iodine $/ \mathrm{g}$, and the peroxide value was not detected. Density and viscosity were 0.92 $\mathrm{mg} / \mathrm{ml}$ and 64 centipoises, respectively. The highest contents of fatty acids were linoleic $(C 18: 2 n 6)$, oleic (C18:1n9) and palmitic (C16:0). Eicosapentaenoic acid (C20:5n3, EPA) and docosahexaenoic acid (C22:6n3, DHA) account for $9 \%$ of the total. The content of astaxanthin was $2.72 \mathrm{mg} / \mathrm{g}$ dry weight. The pigmented oil is a dietary source of nutrients with high value such as astaxanthin.

KEY-WORDS: Astaxanthin - Fatty acids - Gas chromatography - Lactic fermentation - Oil - Shrimp heads.

\section{INTRODUCCIÓN}

La acuicultura, como la mayoría de las actividades productivas, genera gran cantidad de subproductos y residuos. El camarón es, en valor, el principal producto pesquero comercializado y representa el $16.5 \%$ de la producción mundial, de la cual el $40 \%$ es de producción acuícola (FAO, 2006). Esta actividad en México, ha tenido un periodo de expansión que inició a finales del siglo pasado. El Estado de Sonora, al Noroeste del país, es el principal productor y en 2008 se reportó una producción de más de 81,000 toneladas (SAGARPA, 2008). De este volumen solamente se aprovecha el $65 \%$ del crustáceo para consumo humano, el resto corresponde al exoesqueleto y el cefalotórax (López-Cervantes et al., 2006b). Estos residuos generalmente son depositados, en el mejor de los casos, en vertederos municipales, convirtiéndose así en un foco de infección debido a que son susceptibles al deterioro por microorganismos.

Una alternativa para la conservación y aprovechamiento de estos residuos es la fermentación ácido láctica, la cual permite separar los tres principales componentes: quitina, proteínas y lípidos. En la fracción lipídica se encuentran pigmentos de tipo carotenoide, principalmente astaxantina y ácidos grasos insaturados (Armenta-López et al., 2002; López-Cervantes et al., 2006a; No et al., 1989). 
La astaxantina pertenece a la familia de las xantofilas, compuestos oxigenados derivados de los carotenoides. Presenta un color rojo-anaranjado por lo que se utiliza para pigmentar el salmón, truchas, crustáceos y algunas aves. Además, posee mayor actividad antioxidante que la vitamina $\mathrm{E}$, debido a esto se utiliza en productos nutracéuticos y cosméticos (Higuera-Ciapara et al., 2006; Del Río et al., 2005).

Se han realizado diversos estudios para extraer astaxantina a partir de residuos de crustáceos fermentados y no fermentados en combinación con solventes orgánicos (Gimeno et al., 2007; Armenta et al., 2002; Sachindra et al., 2007), mediante metodologías enzimáticas (Armenta y Guerrero-Legarreta, 2009; Babu et al., 2008; Lee et al., 1999), extracción con aceites de origen vegetal y marino (Chen y Meyers, 1984; Sachindra y Mahendrakar, 2005; Handayani et al., 2008). Además, se ha reportado la obtención de una pasta lipídica rica en astaxantina de los residuos fermentados de camarón (López-Cervantes et al., 2010). En algunas investigaciones recientes se presenta la extracción con fluidos supercríticos como una técnica novedosa para la obtención de la astaxantina de algas, levaduras y residuos de crustáceos (Valderrama et al., 2003; Félix-Valenzuela et al., 2001; Passos et al., 2006). Para nuestro conocimiento, no hay estudios relacionados con la calidad de aceites obtenidos por fermentación láctica de los residuos de camarón. Por lo tanto, el presente trabajo ha sido realizado para investigar la calidad fisicoquímica, perfil de ácidos grasos y contenido de astaxantina del aceite pigmentado de camarón. Nuestra hipótesis fue que este aceite tiene utilidad como suplemento alimenticio debido a sus nutrientes y origen biológico.

\section{MATERIALES Y MÉTODOS}

\subsection{Materiales}

Los residuos o cabezas de camarón (Litopenaeus vannamel) provenían de fábricas procesadoras y congeladoras ubicadas en el Sur del Estado de Sonora, México. Estos residuos fueron molidos y congelados a $-20^{\circ} \mathrm{C}$, previamente a su utilización. La astaxantina (98\%) y los estándares de ácidos grasos (FAME MIX C8- C22 y PUFA No. 3) fueron obtenidos de Sigma (St. Louis, Missouri, USA) y Supelco (Bellefonte, Pennsylvania, USA). El ácido acético glacial, fenoftaleína, yoduro de potasio, tiosulfato de sodio, ácido bórico, hidróxido de sodio, hexano, ácido sulfúrico y ácido clorhídrico fueron grado reactivo y se compraron a Productos de Monterrey (Monterrey, Nuevo León, México). El sulfato de sodio anhídrido se obtuvo de Fluka (MiIwakee, Wisconsin, USA), mientras que la mezcla de selenio se adquirió de Merck (Dramstadt, Hesse, Alemania). El tolueno y el carbonato de potasio fueron adquiridos de Riedel-de Haën (Hannover, Baja Sajonia, Alemania).

\subsection{Obtención del aceite}

El proceso de fermentación y la separación de las fases fue el propuesto por Bueno-Solano et al. (2009) y Sánchez-Machado et al. (2008). Específicamente, una porción de los residuos, homogéneamente mezclados, se colocó en un recipiente de 1 litro y se mezcló con 10\% (peso/peso) de azúcar de caña y $5 \%$ (volumen/peso) de inóculo comercial (densidad óptica de 1.7). La mezcla se agitó y se incubó en baño de agua a $30^{\circ} \mathrm{C}$ durante 36 horas. Posteriormente, el fermentado fue centrifugado $\left(1,250 \mathrm{rpm}, 5^{\circ} \mathrm{C}, 15 \mathrm{~min}\right)$ para separar la fracción quitinosa (sedimentada en el fondo), la acuosa intermedia (hidrolizado proteico) y la lipídica como sobrenadante, las que fueron aisladas manualmente. Por último, la fase lipídica fue centrifugada $\left(2,400 \mathrm{rpm}, 5^{\circ} \mathrm{C}, 15 \mathrm{~min}\right)$, observándose una pasta de color marrón en el fondo y en la superficie un aceite viscoso rojo oscuro. A este último se le denominó aceite pigmentado, se recolectó y almacenó en la oscuridad. Las muestras que se analizaron procedían de tres lotes de fermentación (M1, M2 y M3).

\subsection{Análisis proximal}

Los contenidos de humedad, proteínas, cenizas y lípidos totales se determinaron mediante los métodos descritos por la AOAC (1984).

\subsection{Parámetros físico-químicos}

Siguiendo la metodología de la AOCS (1989) se determinó índice de peróxido (cd 8-53), índice de saponificación (cd 3-25) e índice de yodo (cd 1-25). Para determinar la viscosidad se siguió el protocolo de Amado y Mora (2006), se midió a diferentes velocidades de corte $(10,20,30,50$ y 60 revoluciones por minuto) en un viscosímetro DV-E (BROOKFIELD, Middleboro, MA, USA) equipado con el usillo No. 1. La densidad fue estimada con un densímetro (F. Mántey, México, D.F.) a $25^{\circ} \mathrm{C}$ (Amado y Mora 2006; Graciani 2006). Específicamente, se colocó en una probeta $500 \mathrm{ml}$ del aceite y después de estandarizar la temperatura a $25^{\circ} \mathrm{C}$ se sumergió el densímetro hasta que su flotamiento se estabilizó.

\subsection{Composición de ácidos grasos}

El perfil de ácidos grasos (AG) se determinó mediante cromatografía de gases, cuantificándolos en forma de metilésteres. Los AG se metilaron siguiendo el procedimiento de Sánchez-Machado et al. (2009), y se analizaron 3 muestras en forma independiente por triplicado. Específicamente, se pesaron $50 \mathrm{mg}$ de aceite en un tubo con rosca, se adicionaron $2 \mathrm{ml}$ de tolueno y $3 \mathrm{ml}$ de $\mathrm{HCl}$ metanólico al $5 \%$ recién preparado. Esta mezcla se agitó en el vortex y se calentó por 2 horas a $70^{\circ} \mathrm{C}$ en baño de agua. Después se enfrió a temperatura am- 
biente, y se añadieron $3 \mathrm{ml}$ de $\mathrm{K}_{2} \mathrm{CO}_{3}$ al $6 \%$ y $2 \mathrm{ml}$ de tolueno, seguido de agitación en el vortex. La mezcla se centrifugó a 2,400 rpm por $5 \mathrm{~min}$, después se separó la fase orgánica (superior) y se secó con $\mathrm{Na}_{2} \mathrm{SO}_{4}$ anhídrido. El cromatógrafo de gases (VARIAN 3800, Melbourne, Victoria, Australia) esta equipado con un detector de ionización de flama (FID) y una columna capilar CP-Sil $88(60 \mathrm{~m} \times$ $0.25 \mathrm{~mm}$, VARIAN). Las condiciones de operación fueron: volumen de inyección, $1 \mu \mathrm{l}$; temperatura del inyector, $220^{\circ} \mathrm{C}$; gas portador, helio; temperatura del detector, $235^{\circ} \mathrm{C}$. El gradiente de temperatura en el horno de la columna inicia a $120^{\circ} \mathrm{C}$ por $1 \mathrm{~min}$, seguido por incrementos de $3^{\circ} \mathrm{C} / \mathrm{min}$ hasta $170^{\circ} \mathrm{C}$, se mantiene en ésta por 1 min para finalmente ascender a $235^{\circ} \mathrm{C}$ con incrementos de $6^{\circ} \mathrm{C} / \mathrm{min}$, permaneciendo finalmente $5 \mathrm{~min}$ a $235^{\circ} \mathrm{C}$. Los picos individuales de los AG se identificaron por comparación con los tiempos de retención de AG del estándar de referencia PUFA No 3. La abundancia relativa de cada AG se estima en proporción al área de pico identificado. Para la cuantificación se construyó una curva de calibración externa a partir del estándar FAME MIX C8-C22.

\subsection{Cuantificación de astaxantina}

La preparación de la muestra se llevó a cabo según el método propuesto por López-Cervantes et al. (2006a). Se pesó $0.1 \mathrm{~g}$ de muestra y se disolvió en $5 \mathrm{ml}$ de metanol, se agitó la mezcla en vortex por 1 min y luego se sonificó por 5 min, por último se centrifugó a $2400 \mathrm{rpm}$ durante $10 \mathrm{~min}$. El extracto fue diluido y se cuantificó la astaxantina en un espectrofotómetro (Genesys 10 UV Scanning, Madison, Wisconsin, USA) a $476 \mathrm{~nm}$. Para calcular la concentración se construyó una curva de calibración en base a una concentración conocida de astaxantina (Sigma, St. Louis, Missouri, USA). La ecuación de la recta $y=0.0469 x+0.0679$ muestra un coeficiente de correlación ( $r^{2}$ igual a 0.9995) apropiado. Las muestras se analizaron por triplicado.

\subsection{Análisis estadístico}

Los datos obtenidos fueron tratados con el paquete estadístico SPSS versión 12, calculándose las medias y la desviación estándar de las repeticiones de cada uno de los análisis. Además, se realizó un análisis de varianza y la prueba de Tukey para comparar los resultados de los tres lotes.

\section{RESULTADOS Y DISCUSIÓN}

\subsection{Composición proximal del aceite}

Cabe señalar que no se encontraron diferencias significativas entre los tres lotes de fermentación para el análisis proximal. El contenido promedio de lípidos en las muestras supera el $95 \%$, seguido por las proteínas $(2 \%)$, la humedad $(2 \%)$ y al final en menor proporción se encuentran las cenizas $(0.25 \%)$ (Tabla 1). Según la norma mexicana NMXF-223-1985, la humedad no debe exceder $0.05 \%$ en aceites comestibles. La humedad en el aceite se debe a la ineficiencia en la separación manual de las fracciones del fermentado. Para solucionar esto, se ha propuesto complementar la mejora de la separación con un proceso por filtración con agentes desecantes. Sin embargo, ésta situación no se presenta en los aceites extraídos por medio de solventes debido a las diferencias de polaridad con el agua (Baylei, 1984).

Según López-Cervantes et al. (2010) la fracción lipídica obtenida de los fermentados de residuos de camarón posee $5.11 \%$ de cenizas, valor muy por encima del encontrado en el aceite pigmentado. Las sales de calcio y otros minerales se solubilizan durante la fermentación ocasionando que pequeñas cantidades de los mismos queden depositados dentro del tejido graso (Armenta et al., 2002).

Asimismo, el contenido de proteína en el aceite pigmentado puede estar relacionado con la humedad, ya que ésta se caracteriza por contener proteínas hidrolizadas. Bueno-Solano et al. (2009) reportan que la fracción acuosa que resulta de la fermentación es rica en péptidos y aminoácidos libres (8.43\%). Además, Armenta y Guerrero-Legarreta (2009) mencionan la presencia de carotenoproteínas en la fracción lipídica como resultado de la fermentación.

Según Armenta et al. (2002) el contenido de lípidos en el cefalotórax de camarón es $4.63 \%$. Sin embargo, López-Cervantes et al. (2010) reportaron que la fracción lipídica del fermentado de cefalotórax de camarón contiene $42.67 \%$ de lípidos totales. El aceite pigmentado de camarón contiene más del doble de lípidos que la fracción lipídica obtenida por la fermentación de los residuos. Esto se atribu-

Tabla 1

Análisis proximal (\%) del aceite pigmentado de camarón ${ }^{1}$

\begin{tabular}{ccccc}
\hline Lote & Humedad & Cenizas & Proteínas & Lípidos \\
\hline M1 & $2.06 \pm 0.04^{\mathrm{a}}$ & $0.27 \pm 0.04^{\mathrm{a}}$ & $2.03 \pm 0.10^{\mathrm{a}}$ & $95.89 \pm 0.46^{\mathrm{a}}$ \\
M2 & $2.04 \pm 0.04^{\mathrm{a}}$ & $0.25 \pm 0.03^{\mathrm{a}}$ & $2.01 \pm 0.04^{\mathrm{a}}$ & $95.48 \pm 0.62^{\mathrm{a}}$ \\
M3 & $2.03 \pm 0.06^{\mathrm{a}}$ & $0.25 \pm 0.05^{\mathrm{a}}$ & $2.03 \pm 0.08^{\mathrm{a}}$ & $95.73 \pm 0.30^{\mathrm{a}}$ \\
\hline
\end{tabular}

${ }^{1}$ Promedio de tres repeticiones \pm desviación estándar. Letras diferentes en cada columna indican diferencias significativas (Tukey, $\mathrm{p}<0.05$ ). 
ye a que la centrifugación ha permitido separar a algunos componentes líquidos y sólidos inmiscibles en el aceite tales como agua, proteínas y minerales. El rendimiento de extracción del aceite pigmentado por centrifugación es de aproximadamente el $20 \%$ con respecto a la fracción lipídica.

\subsection{Parámetros físico-químicos}

En la Tabla 2 se muestran los resultados para cada una de las tres muestras, se destaca que solo se encontró diferencia significativa en los resultados del índice de saponificación. Tradicionalmente, los aceites de origen vegetal o de pescado son extraídos por métodos mecánicos o por solventes, mientras que el aceite pigmentado de camarón se origina a partir de un proceso fermentativo complementado con la separación por centrifugación.

El índice de saponificación mide el peso molecular medio de los ácidos grasos libres y combinados que existen en las sustancias grasas. Por lo general, los aceites de origen marino tienen índices de saponificación que oscilan entre 180 y 190 mg de $\mathrm{KOH}$ por gramo de muestra. Los índices promedios entre las muestras oscilan en ese rango. Aun con las diferencias observadas, atribuibles al proceso fermentativo y a las características bioquímicas de los residuos de camarón, el índice de saponificación de las muestras es propio de un aceite de origen marino (Bailey, 1984).

El índice de yodo es una medida del grado de insaturación de los componentes de una grasa y se relaciona con su peso molecular promedio. Datos de Allinger y Cava (1972) muestran que el índice de yodo en grasas de origen marino se encuentra por encima de 100 centigramos de yodo absorbidos por gramo de muestra. Las muestras de aceite pigmentado de camarón superan este valor (Tabla 2). Lo anterior es un indicativo de la presencia de ácidos grasos monoinsaturados y poliinsaturados. Ortega-Nieblas et al. (2001) mencionan que el índice de yodo tiende a decrecer cuando el aceite sufre un proceso de oxidación, debido a que ésta conduce a una disminución de los ácidos grasos poliinsaturados. Sin embargo, éste proceso es retardado por la presencia de antioxidantes naturales como la astaxantina y tocoferoles (López-Cervantes et al., 2006a).

El índice de peróxido indica la presencia de oxígeno unido a las grasas en forma de peróxido y muestra hasta que punto se ha alterado la grasa. No se detectaron peróxidos en los aceites analizados. La presencia de antioxidantes naturales, como la astaxantina y los ácidos grasos insaturados, son responsables de inhibir a las sustancias oxidantes (Winston et al., 2004). Rao et al. (2007) estudiaron la estabilidad de distintos aceites comestibles adicionados con astaxantina a temperatura ambiente, 70 y $90^{\circ} \mathrm{C}$, y concluyeron que la capacidad antioxidante de éste compuesto decrece el índice de peróxido existente en los aceites y les aporta un efecto protector. El aceite extraído de los residuos de camarón presenta de manera natural a la astaxantina (Tabla 4).

Los valores de densidad promedio del aceite pigmentado de camarón se presentan en la Tabla 2. Bailey (1984) refiere que la densidad del aceite de ballena a $25^{\circ} \mathrm{C}$ está en el rango de 0.91 a $0.92 \mathrm{~g} /$ $\mathrm{ml}$. Igualmente, Huang y Sathivel (2008) reportaron $0.92 \mathrm{mg} / \mathrm{ml}$ para el aceite de residuos de salmón, y ambos valores son similares a los mostrados por el aceite de camarón.

En la Tabla 2 se presenta la viscosidad para las tres muestras de aceite pigmentado. Los valores promedio fueron 64, 64, 64, 65 y 64 centipoises, respectivamente. Esto indica que el aceite pigmentado se comporta como un fluido newtoniano al no variar su viscosidad con respecto a la velocidad de corte. Según Graciani (2006), Ios fluidos monofásicos de bajo peso molecular tienden a presentar ésta característica. Adicionalmente, Sathivel (2005) reporta el mismo comportamiento para el aceite de cabezas de salmón.

\subsection{Composición de ácidos grasos}

Los aceites de origen marino se caracterizan por la presencia de AG insaturados de cadena larga, a diferencia de los aceites vegetales, debido a esta característica se han perfilado como alimentos nutracéuticos. En las muestras de aceite pigmentado obtenido por fermentación de las cabezas de camarón se identificaron 14 AG y solo se encontró diferencia significativa entre los lotes en cuanto al ácido oleico y vacénico (C18:1n7) (Tabla 3).

Los ácidos grasos que se encontraron en mayor cantidad son el linoleico, oleico y palmítico, en orden decreciente, y representan el $65 \%$ del total. Los ácidos grasos en menor proporción son el linolénico (C18:3n3), gadoleico (C20:1n9), araquidóni-

Tabla 2

Caracterización físico-química del aceite pigmentado ${ }^{1}$

\begin{tabular}{cccccc}
\hline Muestra & $\begin{array}{c}\text { I. Saponificación } \\
(\mathbf{m g ~ K O H} / \mathbf{g})\end{array}$ & $\begin{array}{c}\text { I. Yodo } \\
(\mathbf{c g} / \mathbf{g})\end{array}$ & $\begin{array}{c}\text { I. Peróxido } \\
(\mathbf{m e q} / \mathbf{k g})\end{array}$ & $\begin{array}{c}\text { Densidad } \\
(\mathbf{g} / \mathbf{m l})\end{array}$ & $\begin{array}{c}\text { Viscosidad } \\
\text { (cP) }\end{array}$ \\
\hline M1 & $166.22 \pm 6.77^{\mathrm{a}}$ & $139.41 \pm 2.01^{\mathrm{a}}$ & $\mathrm{ND}$ & $0.92^{\mathrm{a}}$ & $64.2 \pm 0.45^{\mathrm{a}}$ \\
M2 & $180.83 \pm 7.80^{\mathrm{ab}}$ & $139.25 \pm 1.40^{\mathrm{a}}$ & $\mathrm{ND}$ & $0.92^{\mathrm{a}}$ & $63.8 \pm 0.84^{\mathrm{a}}$ \\
M3 & $188.81 \pm 2.13^{\mathrm{b}}$ & $140.74 \pm 0.65^{\mathrm{a}}$ & $\mathrm{ND}$ & $0.92^{\mathrm{a}}$ & $64.4 \pm 0.55^{\mathrm{a}}$ \\
\hline
\end{tabular}

${ }^{1}$ Promedio de tres repeticiones \pm desviación estándar. Letras diferentes en cada columna indican diferencias significativas (Tukey, $\mathrm{p}<0.05)$. ND: No detectado. 
Tabla 3

Perfil de ácidos grasos (\% con respecto del total) en el aceite pigmentado ${ }^{1}$

\begin{tabular}{lrrr}
\hline Acido Graso & \multicolumn{1}{c}{ M1 } & \multicolumn{1}{c}{ M2 } & \multicolumn{1}{c}{ M3 } \\
\hline C14:0 & $3.05 \pm 0.13^{\mathrm{a}}$ & $2.94 \pm 0.12^{\mathrm{a}}$ & $3.03 \pm 0.16^{\mathrm{a}}$ \\
C16:0 & $19.27 \pm 0.92^{\mathrm{a}}$ & $19.40 \pm 0.55^{\mathrm{a}}$ & $18.41 \pm 0.80^{\mathrm{a}}$ \\
C16:1n7 & $5.34 \pm 0.08^{\mathrm{a}}$ & $5.17 \pm 0.16^{\mathrm{a}}$ & $5.18 \pm 0.21^{\mathrm{a}}$ \\
C18:0 & $4.26 \pm 0.04^{\mathrm{a}}$ & $4.34 \pm 0.09^{\mathrm{a}}$ & $4.05 \pm 0.03^{\mathrm{a}}$ \\
C18:1n9 & $22.44 \pm 0.22^{\mathrm{ab}}$ & $22.74 \pm 0.24^{\mathrm{b}}$ & $21.97 \pm 0.04^{\mathrm{a}}$ \\
C18:1n7 & $4.98 \pm 0.22^{\mathrm{a}}$ & $5.07 \pm 0.05^{\mathrm{a}}$ & $5.45 \pm 0.04^{\mathrm{b}}$ \\
C18:2n6 & $24.46 \pm 0.40^{\mathrm{a}}$ & $24.51 \pm 0.06^{\mathrm{a}}$ & $24.25 \pm 0.19^{\mathrm{a}}$ \\
C18:3n3 & $1.67 \pm 0.05^{\mathrm{a}}$ & $1.65 \pm 0.00^{\mathrm{a}}$ & $1.63 \pm 0.02^{\mathrm{a}}$ \\
C20:1n9 & $1.17 \pm 0.05^{\mathrm{a}}$ & $1.18 \pm 0.04^{\mathrm{a}}$ & $1.16 \pm 0.06^{\mathrm{a}}$ \\
C20:4n6 & $1.10 \pm 0.02^{\mathrm{a}}$ & $1.11 \pm 0.05^{\mathrm{a}}$ & $1.11 \pm 0.03^{\mathrm{a}}$ \\
C20:4n3 & $1.46 \pm 0.07^{\mathrm{a}}$ & $1.44 \pm 0.05^{\mathrm{a}}$ & $1.54 \pm 0.11^{\mathrm{a}}$ \\
C20:5n3 & $4.85 \pm 0.11^{\mathrm{a}}$ & $4.84 \pm 0.29^{\mathrm{a}}$ & $5.06 \pm 0.26^{\mathrm{a}}$ \\
C22:5n3 & $2.10 \pm 0.21^{\mathrm{a}}$ & $1.98 \pm 0.13^{\mathrm{a}}$ & $2.58 \pm 0.36^{\mathrm{a}}$ \\
C22:6n3 & $3.86 \pm 0.32^{\mathrm{a}}$ & $3.63 \pm 0.26^{\mathrm{a}}$ & $4.85 \pm 0.54^{\mathrm{a}}$ \\
\hline
\end{tabular}

${ }^{1}$ Promedio de tres repeticiones \pm desviación estándar. Letras diferentes en cada columna indican diferencias significativas (Tukey, $\mathrm{p}<0.05$ ).

co (C20:4n6) y eicosatetraenoico (C20:4n3), representando el 5\%. Guillou et al. (1995) reportan que los ácidos oleico y palmítico predominan en el aceite crudo extraído químicamente de los residuos de camarón. Por otro lado, Shahidi y Synowieck (1991) encontraron que el ácido oleico es más abundante en los extractos de residuos de cangrejo sin fermentar. Las variaciones de los resultados pueden atribuirse a la especie de crustáceo, así como a su alimentación.

El contenido de ácidos grasos omega 3 en el aceite pigmentado representa el 14\%. Según Calder (2001), el aceite de pescado contiene entre 15 y $30 \%$ de AG omega 3 . En éste grupo, los ácidos eicosapentaenoico (C20:5n3, EPA) y docosahexaenoico (C22:6n3, DHA) constituyen el 9\%, ambos tienen entre sus funciones bioquímicas el ser precursores de eicosanoides, los cuales actúan como mediadores lipídicos el óptimo funcionamiento del sistema inmune (Simopoulus, 1999).

En este estudio, cinco ácidos grasos fueron cuantificados debido a que han sido identificados con anterioridad por diversos autores como esterificantes de la astaxantina en crustáceos (CoralHinostroza y Bjerkeng, 2002; Lin et al., 2005; Takaichi et al., 2003). El contenido de ácido mirístico (C14:0), palmítico, palmitoleico (C16:1n7), esteárico (C18:0) y oleico correspondió a 13.47, 73.92, 22.77, 19.72 y 100.44 (mg/g en base húmeda), respectivamente. Es posible que la astaxantina presente en el aceite pigmentado esté esterificada con alguno de éstos ácidos grasos como resultado de la estabilización que sufre el pigmento durante la fermentación (Armenta et al., 2002).
Tabla 4

Contenido de astaxantina (base seca) en el aceite pigmentado ${ }^{1}$

\begin{tabular}{cc}
\hline Muestra & Astaxantina $(\mathrm{mg} / \mathrm{g})$ \\
\cline { 2 - 2 } M1 & $3.09 \pm 0.00^{\mathrm{c}}$ \\
M2 & $2.38 \pm 0.03^{\mathrm{a}}$ \\
M3 & $2.69 \pm 0.02^{\mathrm{b}}$ \\
\hline
\end{tabular}

${ }^{1}$ Promedio de tres repeticiones \pm desviación estándar. Letras diferentes en cada columna indican diferencias significativas (Tukey, $\mathrm{p}<0.05$ ).

\subsection{Cuantificación de astaxantina}

El contenido del pigmento astaxantina en los aceites se muestra en la Tabla 4. El valor promedio es de $2.72 \mathrm{mg} / \mathrm{g}$ en base seca y se encontró diferencia estadística entre los tres lotes, esto puede asociarse a la cantidad y disponibilidad del pigmento en la dieta de los crustáceos (Armenta et al., 2002). López-Cervantes et al. (2006a) reportaron que la fracción lipídica del fermentado de los residuos de camarón sin centrifugar contiene $2.21 \mathrm{mg}$ de astaxantina por gramo de muestra seca. El aceite extraído con acetona del ensilaje de residuos de camarón (Pandalus borealis) contiene $4.57 \mathrm{mg}$ de pigmento/g, estas diferencias se pueden atribuir al proceso de extracción y a la especie de camarón (Guillou et al., 1995). Según Rao et al. (2007) la estabilización del pigmento en los aceites depende de su composición de ácidos grasos. Como se indicó anteriormente, la fracción lipídica cruda tiene $40 \%$ de lípidos mientras que en el aceite hay $95 \%$, esto favorece la esterificación de la 


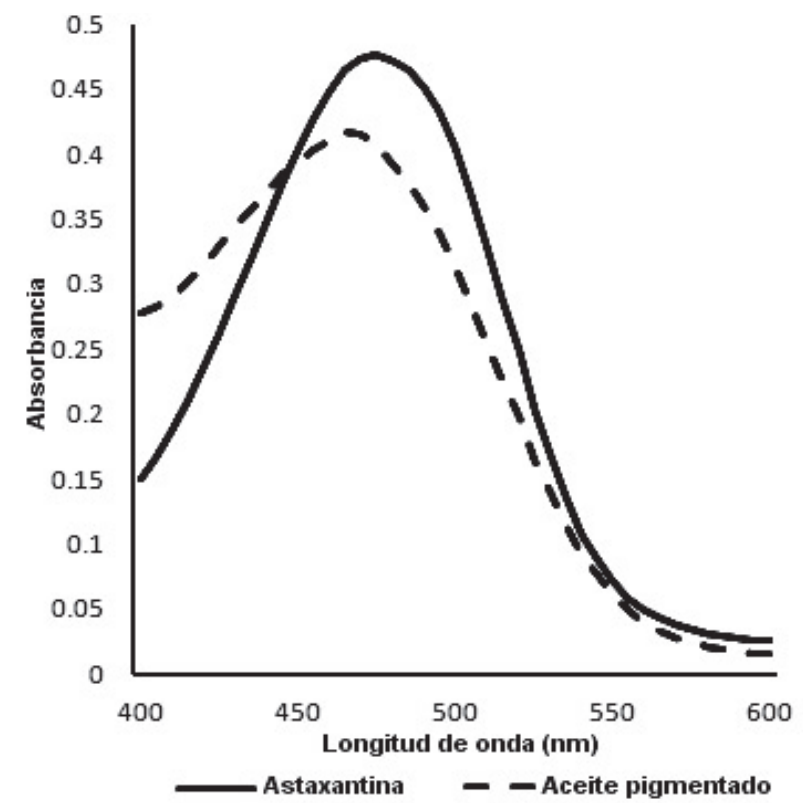

Figura 1

Espectro de absorción de astaxantina y de una muestra de aceite pigmentado de camarón en metanol.

astaxantina y por lo tanto su estabilización (LópezCervantes et al., 2010). En la figura 1, se presenta el espectro de absorción del estándar de astaxantina y el de una muestra de aceite pigmentado.

Sachindra y Mahendrakar (2005) aislaron astaxantina de los residuos de camarón sin fermentar utilizando aceites vegetales y el rendimiento máximo se observó con aceite de girasol (0.026 mg de pigmento por gramo de residuo), lo cual es 100 veces menor que lo encontrado en el aceite pigmentado. También, Fujita et al. (1983) obtuvieron aceite pigmentado de krill con solventes (hexano:etanol, 79:21, v/v) y reportan el contenido de carotenoides totales $(1.08 \mathrm{mg} / \mathrm{g})$. De acuerdo a Omara-Alwala et al. (1985), un aceite con $0.6 \mathrm{mg}$ de astaxantina por gramo tiene un nivel económicamente aceptable para su incorporación en dietas a fin de pigmentar al salmón comercial.

\section{CONCLUSIÓN}

El aceite pigmentado aislado de los residuos de camarón presenta las características generales de un aceite de origen marino y se muestra como una fuente de ácidos grasos insaturados, especialmente EPA y DHA, y astaxantina. Futuras investigaciones se podrían enfocar en la evaluación nutricional del aceite como suplemento para consumo humano y para la elaboración de piensos destinados a la pigmentación de peces y aves.

\section{AGRADECIMIENTOS}

Esta investigación ha sido financiada por el Proyecto FONCICYT número C002-2008-1/ALA - 127 249. El primer autor agradece a CONACYT la beca para estudios de doctorado.

\section{REFERENCIAS}

Allinger NL, Cava MP. 1972. Química orgánica. Ed. Reverté, Barcelona, España.

Amado E, Mora L. 2006. Análisis de la variación de la viscosidad cinemática de un aceite vegetal en función de la temperatura. Bistua 4, 54-56.

AOAC. 1984. Official Methods of Analysis (14 th. Ed.). S. Williams Editor. Association of Official Analytical Chemists Inc. Arlington, Virginia.

AOCS. 1989. Official and Tentative Methods. (3rd. Ed.). W.E. Link Editor. American Oils Chemists Society. Champaign, Illinois, U.S.A.

Armenta RE, Guerrero-Legarreta I, Huerta S. 2002. Extracción de carotenoproteínas a partir de residuos de camarón fermentados. Rev. Mex. Ing. Quí. 1, 49-55.

Armenta RE, Guerrero-Legarreta I. 2009. Amino acid profile and enhancement of the enzymatic hydrolysis of fermented shrimp carotenoproteins. Food Chem. 112, 310-315.

Armenta-López RE, Guerrero I, Huerta S. 2002. Astaxanthin extraction from shrimp waste by lactic fermentation and enzymatic hydrolysis of the carotenoprotein complex. J. Food Sci. 67, 10021006.

Babu CM, Chakrabarti R, Surya SKR. 2008. Enzymatic isolation of carotenoid complex from shrimp head waste and its use as a source of carotenoids. LWT 41, 227-235.

Bailey AE. 1984. Grasas y aceites industriales. Ed. Reverté, Barcelona, España.

Bueno-Solano C, López-Cervantes J, Campas-Baypoli ON, Lauterio-García R, Adan-Bante NP, SánchezMachado DI. 2009. Chemical and biological characteristics of protein hydrolysates from fermented shrimp by-products. Food Chem. 112, 671-675.

Calder PC. 2001. More good news about fish oil. Nutr. $17,158-159$

Chen HM, Meyers SP. 1984. A rapid quantitative method for determination of astaxanthin pigment concentration in oil extracts. J. Am. Oil Chem. Soc. 61, 1045-1047.

Coral-Hinostroza GN, Bjerkeng B. 2002. Astaxanthin from the red crab langostilla (Pleuroncodes planipes): optical R/S isomers and fatty acids moieties of astaxanthin esters. Comp. Biochem. Physiol. Part B 133, 437-444.

Del Río E, Acién FG, García-Malea MC, Rivas J, Molina-Grima E, Guerrero MG. 2005. Efficient onestep production of astaxanthin by the microalga Haematococcus pluvialis in continuous culture. Biotechnol. Bioeng. 91, 808-815.

FAO. 2006. El estado mundial de la pesca y la acuicultura. Departamento de pesca y acuicultura. Roma, Italia.

Felix-Valenzuela L, Higuera-Ciapara I, GoycooleaValencia F. 2001. Supercritical $\mathrm{CO}_{2}$ /ethanol extraction of astaxanthin from blue crab (Callinectes sapidus) shell waste. J. Food Process Eng. 24, 101-112.

Fujita T, Satake M, Hikichi S, Takeda M, Shimeno S, Kuwabara H, Miki W, Yamaguchi K, Konosu S. 1983. Pigmented of cultured yellowtail with krill oil. Bull. Japan. Soc. Sci. Fish. 49, 1595-1600.

Gimeno M, Ramírez-Hernández JY, Martínez-lbarra C, Pacheco N, García-Arrazola R, Bárzana E, Shirai K. 2007. One-solvent extraction of astaxanthin from lactic acid fermented shrimp wastes. J. Agric. Food Chem. 55, 10345-10350. 
Graciani CE. 2006. Los aceites y grasas: composición y propiedades. Ed. Madrid Vicente y Mundi-Prensa, Madrid, España.

Guillou A, Khalil M, Adambounou L. 1995. Effects of silage preservation on astaxanthin forms and fatty acid profiles of processed shrimp (Pandalus borealis) waste. Aquacult. 130, 351-360.

Handayani AD, Sutrisno, Indraswati N, Ismadji S. 2008. Extraction of astaxanthin from giant tiger (Panaeus monodon) shrimp waste using palm oil: studies of extraction kinetics and thermodynamic. Biores. Technol. 99, 4414-4419.

Higuera-Ciapara I, Félix-Valenzuela L, Goycoolea FM. 2006. Astaxanthin: a review of its chemistry and applications. Crit. Rev. Food Sci. Nutr. 46, 185-196.

Huang J, Sathivel S. 2008. Thermal and rheological properties and the effects of temperature on the viscosity and oxidation rate of unpurified salmon oil. J. Food Eng. 89, 105-111.

Lee SH, Roh SK, Park KH, Yoon KR. 1999. Effective extraction of astaxanthin pigment from shrimp using proteolytic enzymes. Biotechnol. Bioprocess Eng. 4, 199-204.

Lin WC, Chien JT, Chen BH. 2005. Determination of carotenoids in spear shrimp shells (Parapenaeopsis hardwickil) by liquid chromatography. J. Agric. Food Chem. 53, 5144-5149.

López-Cervantes J, Adan-Bante NP, Sánchez-Machado DI. 2010. Separation and biochemical characterization of the products from fermented shrimp wastes, en Le Bihan E Sea by-products as a real material: new ways of application. Transworld Research Network, Kerala, India, 117-132.

López-Cervantes J, Sánchez-Machado DI, GutiérrezCoronado MA, Rios-Vazquez NJ. 2006a. Quantification of astaxanthin in shrimp waste hydrolysate by HPLC. Biomed. Chromatogr. 20, 981-984.

López-Cervantes J, Sánchez-Machado DI, RosasRodríguez JA. 2006b. Analysis of free amino acids in fermented shrimp waste by high-performance liquid chromatography. J. Chromatogr. A 1105, 106-110.

NMX-F-223-1985. Norma oficial mexicana que establece las especificaciones mínimas de calidad que debe cumplir el producto denominado "aceite vegetal comestible". Diario Oficial de la Federación, México, D.F.

No HK, Meyers SP, Lee KS. 1989. Isolation and characterization of chitin from crawfish shell waste. $J$. Agric. Food Chem. 37, 575-579.

Omara-Alwala TR, Chen HM, Ito $Y$, Simpson HL, Meyers SP. 1985. Carotenoid pigment and fatty acids analyses of crawfish oil extracts. J. Agric. Food Chem. 33, 260-263.
Ortega-Nieblas M, Robles-Burgueño MR, VázquezMoreno L. 2001. Evaluación oxidativa de las muestras de aceites de leguminosas del Desierto de Sonora con aceites de maíz y soja durante su almacenamiento. Grasas y Aceites 52, 355-362.

Passos R, Beirão L, Palavra A, Mendes R, Nobre B, Gouveia L. 2006. Astaxanthin from the yeast Phaffia rhodozyma supercritical carbon dioxide and organic solvents extraction. J. Food Technol. 4, 59-63.

Rao AR, Sarada R, Ravishankar GA. 2007. Stabilization of astaxanthin in edible oils and its use as an antioxidant. J. Sci. Food Agric. 87, 957-965.

Sachindra NM, Bhaskar N, Siddegowda GS, Sathisha AD, Suresh PV. 2007. Recovery of carotenoids from ensilaged shrimp waste. Biores. Technol. 98, 1642-1646.

Sachindra NM, Mahendrakar NS. 2005. Process of optimization for extraction of carotenoids from shrimp waste with vegetable oils. Biores. Technol. 96, 11951200.

SAGARPA. 2008. Anuario Estadístico de Pesca. Secretaria de Agricultura, Ganadería, Desarrollo Rural, Pesca y Alimentación. México, D. F.

Sánchez-Machado DI, Núñez-Gastélum JA, ReyesMoreno C, Ramírez-Wong B, López-Cervantes J. 2009. Nutritional quality of edible parts of Moringa oleifera. Food Anal. Methods 3, 175-180.

Sánchez-Machado DI, López-Cervantes J, MartínezCruz O. 2008. Quantification of organic acids in fermented shrimp waste by HPLC. Food Technol. Biotechnol. 46, 456-460.

Sathivel S. 2005. Thermal and flow properties of oils from salmon heads. J. Am. Oil Chem. Soc. 82, 147-152.

Shahidi F, Synowiecki J. 1991. Isolation and characterization of nutrients and value-added products from snow crab (Chinoecetes opilio). J. Agric. Food Chem. 39, 1527-1532.

Simopoulus AP. 1999. Omega-3 fatty acids in inflammation and immune diseases. J. Am. Coll. Nutr. 21, 495-505.

Takaichi S, Matsui K, Nakamura M, Muramatsu M, Hanada S. 2003. Fatty acids of astaxanthin esters in krill determined by mild mass spectrometry. Comp. Biochem. Physiol. Part B 136, 317-322.

Valderrama JO, Perrut M, Majewski W. 2003. Extraction of astaxantine and phycocyanine from microalgae with supercritical carbón dioxide. J. Chem. Eng. Data 48, 827-830.

Winston GW, Lemaire DGE, Lee RF. 2004. Antioxidants and total oxyradical scavenging capacity during grass shrimp, Palaemonetes pugio, embryogenesis. Comp. Biochem. Physiol. Part C 139, 281-288.

Recibido: 20/10/10 Aceptado: $18 / 1 / 11$ 